\title{
Motion-aided Network SLAM
}

\author{
Joseph Djugash, and Sanjiv Singh \\ The Robotics Institute, Carnegie Mellon University, Pittsburgh, PA 15213, USA \\ \{josephad, ssingh\}@ri.cmu.edu
}

Summary. A key problem in the deployment of sensor networks is that of determining the location of each sensor such that subsequent data gathered can be registered. We would also like the network to provide localization for mobile entities, allowing them to navigate and explore the environment. In this paper, we present a thorough evaluation of our algorithm for localizing and mapping the mobile and stationary nodes in a sparsely connected sensor network using range-only measurements and odometry from the mobile node. Our approach utilizes an Extended Kalman Filter (EKF) in polar space allowing us to model the nonlinearities within the range-only measurements using Gaussian distributions. We demonstrate the effectiveness of our approach using extensive real-world experiments with sparse connectivity and little to no prior information about the node locations.

\section{Introduction}

The growing trend of wireless communication and sensing technologies have emphasized the importance and applicability of sensor networks for a wide variety of application domains. Here we focus on the problem of estimating the node positions of a sensor network given only range data between nodes in the network. Previous work have shown that, given sufficient connectivity between the nodes in the network, it is possible to acquire an accurate estimate of the node positions [1, 2]. However, existing strategies assume fairly simplistic scenarios, where the nodes operate within a large open area with little impedance to their measurements. In contrast, we explore more challenging and realistic scenarios, where the network is sparsely connected, has noisy measurements and has mobile nodes.

In this article, we present a comprehensive method to solve the network SLAM problem that is robust to problems of noisy, sparse and nonlinear measurements. We present experimental results on data that are particularly challenging to range-only estimation due to the amount of measurement noise, multi-path and outlier measurements present in the system. The experiments 
were conducted outdoors, with the nodes distributed around several buildings with no clear line-of-sight between many of the nodes.

The experiments address two distinct cases of network SLAM. First, the "static" network mapping problem is tackled. Here, it is assumed that the nodes are sparsely connected and remain stationary. The particular challenge in this case is to accurately model the nonlinear uncertainty distributions that arise while estimating a sparsely connected node's position in the presence of noise (such as multi-path or other outlier measurements). Next, we extend the static network mapping problem to deal with moving nodes. This general problem of localizing a network of both stationary and mobile nodes is called the network SLAM problem. The addition of mobile nodes provides further constraints to overcome the ambiguities due to sparse connectivity between the nodes. However, the challenge here is to actively fold in the measurements and noisy odometry from the mobile node while maintaining an accurate estimate of the nonlinear uncertainty distributions of all the nodes' positions. We show through our experiments that the proposed method is robust, reliable and accurate even in challenging scenarios.

\section{Related Work}

In sensor networks the problem of mapping the locations of all the nodes in the network is also known as self-calibration or self-localization. Most sensor networks are capable of measuring relative bearing, range or in some cases both range and bearing between nodes within the environment. Of particular interest to us are those that use range to localize the network. For instance, the RADAR system, developed by Bahl and Padmanabhan, utilizes signal strength of packets in the commonly available $802.11 \mathrm{~b}$ wireless networks for localization of network devices [3]. However, signal strength measurements are often erratic and can be affected by slight changes in the environment. Alternately, fixed ultrasound emitters and embedded receivers have also been used to measure range between nodes in a network $[4,5,1]$. Our system utilizes a new commercially available ranging radio system, the nanoLOC ranging system from Nanotron Technologies [6]. These sensors use radio frequency (RF) signals to measure range between two nodes in the network.

Some of the early work in localizing a sensor network with range-only information relied on solving a least-squares optimization problem. Methods such as Multi-dimensional Scaling (MDS) provide a good solution if the network is fully connected [7]. For a less connected network with sufficient connections to provide "rigidity" to the network, it is still possible to determine the map of the network. Moore et al. introduced the idea of the robust quadrilaterals as a way to avoid ambiguities in the solution [8]. In practice, however, rigidity is not easy to achieve a high degree of connectivity between nodes of the network. 
While most research in sensor networks have focused on static nodes (network localization), work in SLAM has focused on the incorporation of motion from mobile robots into the estimation of static and mobile nodes. Olson et al. presented an EKF-based SLAM algorithm that reliably dealt with noisy measurements and required no priori information [9]. Their method utilizes an initial pre-filtering step to approximately locate the landmarks/nodes thus making the linearization feasible. However, the performance of the pre-filter is highly dependent on its input data. Previously, we have presented a method based on an EKF that jointly estimates location of the static and mobile nodes $[1,2,10]$. But, the experiments presented in these works were limited due to their assumption that the nodes was deployed within an open area with high connectivity between them and that there is little multi-path/outlier measurements. In most applications, it is rare that a sensor network can be deployed within an open area where line-of-sight between the nodes can be guaranteed or that the sensor measurements contain minimal erroneous measurements. In this article we demonstrate that the proposed approach, designed to provide a better approximation of the nonlinear measurement distributions, is better suited to deal with sparsely connected networks deployed within an obstacle filled environment (which introduce multi-path and other sources of noise).

\section{Technical Approach}

We model the network localization problem as a linear dynamical system. At each time step, $t$, the state of node $i$ is represented by $X_{i, t}=\left[c_{i}^{x}, c_{i}^{y}, r_{i}, \theta_{i}\right]^{T}$. Each node's estimate is represented in a polar coordinates [10], where $\left(c_{i}^{x}, c_{i}^{y}\right)$ are the center of the polar coordinate frame and $\left(r_{i}, \theta_{i}\right)$ are the corresponding range and angle values. The use of this parameterization derives motivation from the polar coordinate system, where annuli, crescents and other ring-like shapes can be easily modeled. In addition, for each mobile node within the system, an additional term that represents the current heading of the node, $\phi_{i}$, is also maintained within the state. The complete state vector at time $t$ is represented as:

$$
X_{t}=\left[X_{1, t}, \phi_{1}, \ldots, X_{M, t}, \phi_{M}, X_{M+1, t}, X_{M+2, t}, \ldots, X_{N, t}\right]^{T} .
$$

where $M$ is the number of mobile nodes and $N$ is the total number of nodes. At each time step, we get some set of motion and range observations, $u_{t}$ and $z_{t}$ respectively. The belief state at time $t$ is defined as $p\left(X_{t} \mid z_{1: t}, u_{1: t}\right)$. Our filtering algorithm iteratively computes the belief state at time $t+1$ using the previous belief state at time $t$. Specifically, in our implementation the belief state is represented by a mean vector $\mu_{t}$ and a covariance matrix $\Sigma_{t}$, and it is computed using an Extended Kalman Filter (EKF). 


\subsection{Measurement Model}

When two nodes, $i$ and $j$, are within a given range and sensor F.O.V. to each other, a range observation is generated which is represented by, $z_{t}^{i, j}$. This observation depends on the position of the two nodes $i$ and $j$ :

$$
\begin{gathered}
z_{t}^{i, j}=\widehat{z}_{t}^{i, j}\left(X_{i, t}, X_{j, t}\right)+\delta . \\
\widehat{z}_{t}^{i, j}=\sqrt{\left(m_{i, t}^{x}-m_{j, t}^{x}\right)^{2}+\left(m_{i, t}^{y}-m_{j, t}^{y}\right)^{2}} . \\
m_{k, t}^{x}=c_{k, t}^{x}+r_{k, t} \cdot \cos \left(\theta_{k, t}\right) . \\
m_{k, t}^{y}=c_{k, t}^{y}+r_{k, t} \cdot \sin \left(\theta_{k, t}\right) .
\end{gathered}
$$

where $\delta$ is zero-mean Gaussian noise and $\left(m_{k, t}^{x}, m_{k, t}^{y}\right)$ is the projection of the estimate for node $k$ from the polar parameterization into Cartesian xy-space. The belief state is then conditioned on the observations of the current time step by computing:

$$
\begin{gathered}
p\left(X_{t+1} \mid z_{1: t+1}, u_{1: t+1}\right)=\eta p\left(X_{t+1} \mid z_{1: t}, u_{1: t+1}\right) \cdot p\left(z_{t+1} \mid X_{t+1}\right) . \\
p\left(z_{t+1} \mid X_{t+1}\right)=\prod_{k} p\left(z_{t+1}^{k} \mid X_{i \in g\left(z_{t+1}^{k}\right), t+1}\right) .
\end{gathered}
$$

where $\eta$ is the normalization constant. The second term in the r.h.s of Eq.2 is the likelihood of the current observations. Eq.3 shows how this likelihood can be decomposed under the assumption that observations are independent given the locations of the nodes that made the observation. Note that each observation depends only upon the locations of the nodes in the set $g\left(z_{t+1}^{k}\right)$, which is the set of nodes that made the observation, and not the joint state vector. The range observations are augmented into the belief state by multiplying into the belief state a likelihood for each observation.

Upon the first observation of a particular node, the true distribution of the node is best represented as an annulus, see Figure 1(a). While an annulus is extremely non-Gaussian and difficult to model within the Cartesian xy-space, using the polar parameterization it is possible to approximate the annulus by an elongated Gaussian in polar coordinates ( $r \theta$-space). This Gaussian approximation is given an arbitrary mean in $\theta$ (within the range $[0,2 \pi)$ ) with a large variance term, such that the probability along the $\theta$ dimension is near uniform, see Figure 1(b). Figure 1(c) shows the Gaussian ellipse (blue ellipse) overlaid on top of the true distribution (green shaded rectangle) in polar coordinates. By using this polar parameterization, a simple ellipse in polar coordinates transforms into an nonlinear annulus when projected into the xy-space. It must also be noted that the elongated ellipse in the polar coordinate extends past the range of the true distribution. This extended tail of the Gaussian ellipse, when projected into the xy-space appears curled up within the estimated annulus, as can be seen in Figure 1(b). 


\subsection{Multi-Hypothesis Filter}

Thus far, we have assumed an unimodal Gaussian model, capable of approximating the non-linearities within single range observations. We have also presented a probabilistic filtering method that is well suited for an EKF-based network localization system. While this approach deals with non-linearities of an annulus, it fails to adequately deal with the multi-modal distribution of the system ((Figure 1(d))). Thus, whenever an annulus is split into separate modes, we simply duplicate the filter and adjust the mean of each filter to represent the two distinct intersection points. Then, by performing a measurement update using the new mean, we are able to appropriately update the covariance terms within the filter. The simple case of splitting a single annulus into two separate modes given a new range obser-
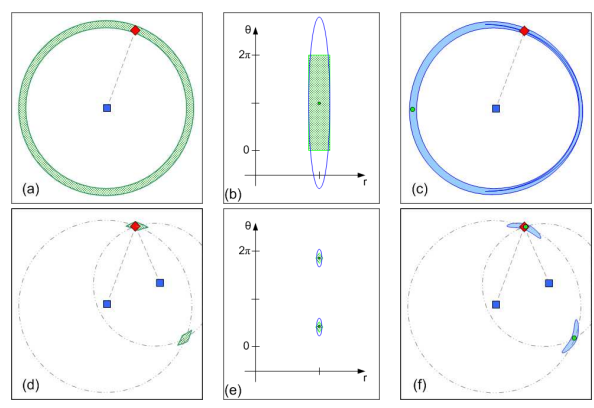

Fig. 1. Blue squares represent observing nodes, whose location is known. Red diamonds represent the true location of the observed node, whose position is being estimated and green circles represent the mean(s) for each mode of the estimated node. Green shaded regions represent the true uncertainty distribution and blue ellipses represent the estimated uncertainty distribution. The dashed gray lines and circles represent the observed range measurements. (a-c) Shows various representation of the annulus-like distribution. (df) Shows various representation of the dual modal "flip-ambiguous" distribution. vation is depicted in Figure 1(d-f).

Given an annulus-like prior distribution, a new range observation that intersects the annulus at two distinct locations leads to a multi-modal distribution with two distinct modes (peaks/local maxima in the distribution). We refer to this as the flip ambiguity in range-only estimation tasks. These multi-modal distribution can be modeled using separate filters/hypotheses for each mode. To elaborate, whenever an annulus is split into separate modes, we simply duplicate the filter and adjust the mean of each hypothesis to represent the two distinct intersection points. It should be noted here that this duplication only occurs if the new measurement is "novel", compared to the initial measurement that initialized the robot to an annulus-like distribution. Novelty of measurements, in this case, is directly correlated to the difference in the locations of the stationary nodes making the two range observations. Thus, if the distance between the node that made the new observation and the original observation is larger than a threshold, the new observation is used to generate a hypothesis. If the distance between the two nodes is less than the threshold, the measurement is used only to perform an EKF measurement update on the existing hypothesis.

Upon duplicating the filter and creating a second hypothesis, it is necessary to adjust the mean of both the hypotheses. The new mean for each of 
the two hypotheses are calculated by triangulation, using the locations of the two stationary nodes that made the observation. Then, by performing a measurement update using the new mean, we are able to appropriately update the covariance terms within the filter. The simple case of splitting a single annulus into two separate modes given a new range observation is shown in Figure 1(d) and (e). Figure 1(f) shows the Gaussian ellipses (blue ellipses) for the dual-modes overlaid on top of the true distribution (green shaded rectangles) in polar coordinates. The mean of the two modes can be determined easily using triangulation, given the location of the two observing nodes, as described in [11]. At the end of each update, we check the (normalized) likelihood of each hypothesis, given all the measurements, and retain hypotheses with likelihoods above a certain threshold (relative to the likelihoods of all existing hypotheses). Additionally, in our implementation, we remove any duplicate hypotheses. A hypothesis is considered duplicate, when it has a mean and covariance similar to another hypothesis. This can be checked using a distribution comparison metric such as the Kullback-Leibler distance (KLdistance). Finally, it should be noted here that in the localization case, the hypothesis count for the system will be never greater than two. Thus, implementing a multi-hypothesis filter is fairly straight forward and efficient.

The ROP parameterization and multi-hypothesis filter proposed here are design to accurately represent the nonlinear distributions that are generated by range-only observations. However, it is important to remember that while the distributions generated by the parameterization is still a linearized version of the true distribution. In other words, the proposed method, while capable of more accurately representing the nonlinear distributions (such as an annulus or crescent), still uses a Gaussian distribution to represent the distribution. It is for this reason that when creating a second hypothesis, the mean of both the hypotheses need to be adjusted. The adjustment, usually only in the $\theta_{t}^{r}$ parameter, highlights the point around which the linearization takes place. Failing to properly adjust the means of the two hypotheses could cause the filter to take longer to converge or even diverge. While this drawback has little effect in the performance of the filter when addressing the localization problem, we will tackle other more challenging problems in the next few chapters where special care needs to be taken to avoid the linearization effects to drastically affect the quality of the filter's estimate.

Furthermore, the proposed approach lends itself to a decentralized implementation that runs separate EKFs on each node in the network. The decentralized approach would require an additional step that gathers the information from neighboring nodes in the network and merges them into each node's own belief using a belief propagation algorithm (we refer the reader to our prior work for further details on this decentralized approach [2]). Additionally, when dealing with a mobile node, care needs to be taken to properly model the motion of the moving node. Whether odometry information is available or if a random walk model is assumed, it needs to be incorporated into the filter correctly [12]. 


\section{Experiments}

We demonstrate the effectiveness of our proposed network mapping algorithm on two types of experiments. The first experiment is the "static" mapping experiment where all the nodes are stationary. Here, we assume the knowledge of a few nodes' true position (ie. anchors) to help provide a rigid reference to the global coordinate frame and to reduce the ambiguities within the system. The second experiment extends the "static" mapping experiment to include a single mobile node to the network. The mobile node moves within the limits of the stationary nodes but rarely has line-of-sight to more than a couple stationary nodes at a time. Here, given the information provided by the mobile node, we show that it is possible to accurately estimate the nodes' position with even fewer anchors.

\subsection{Experimental Setup}

In our experiments we deployed the nodes in an outdoor environment between and around several buildings. Figure 2 shows the floor plan of the environment where the nodes were deployed. The nodes have a maximum range of $120 \mathrm{~m}$ in an open area with line-of-sight (LOS), however, in our experimental environment their maximum range was limited due to the occlusions and obstacles within the environment. However, the nodes provide range measurements even through some obstacles like thin walls (usually noisier than LOS measurements). The presence of such "unmodeled" noise in our measurements introduces an additional challenge to the network SLAM problem. Our experiments were conducted using an autonomous wheelchair equipped with a ranging radio and wheel encoders. The robot was also equipped with a laser scanner to enable it perform simple obstacle avoidance and gather laser scan information about the environment. In addition to ranging radio equipped on the mobile robot, several other stationary ranging radio nodes were arbitrarily deployed within the environment. The connectivity among a network of nodes within the environment, which directly corresponds to the ability to range between a pair of connect nodes, is shown in Figure 3 .

\subsection{Static Mapping}

In most real-world applications where pre-deployed infrastructures are available, it is often the case that the infrastructure is already operational before any mobile agent (such as a robot) enters the environment. In these cases, it is desirable if the pre-deployed nodes can themselves begin to solve the network SLAM problem immediately after their initial deployment. Figure 3 presents the results of our proposed method on a static network of nodes without the assistance of any mobile agents. The network consists of 16 nodes that are sparsely connected due to the obstacles in the environment. Additionally, 


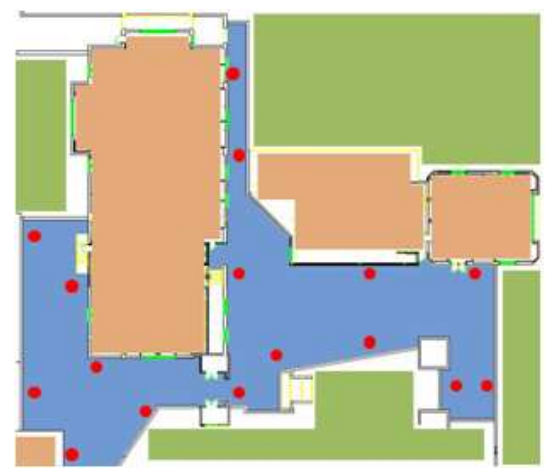

Fig. 2. The blue shaded region indicate the free-space and the red dots indicate the locations of the stationary nodes placed within the environment. The size of the workspace is $55 \mathrm{~m}$ $\mathrm{x} 70 \mathrm{~m}$.

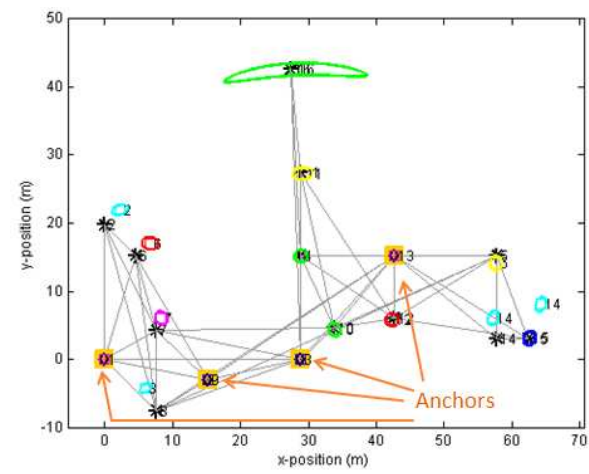

Fig. 3. Estimated node positions for the static mapping scenario. The nodes are plotted with their estimate uncertainties. The true positions of the four anchors nodes are known a priori to provide rigidity to the solution.

some of the range measurements are extremely noisy due to environmental effects such as multi-path.

A particular challenge with static network mapping in sparse networks is determining if a given measurement is either a good, multi-path, non-LOS (through thin obstacles) or outlier measurement and then dealing with it properly. A standard measurement gating technique, chi-square gate, combined with the proposed polar parameterization is employed to reduce the effects of such noisy data on the filter's estimate. As can be seen from our results, the proposed approach was able to accurately reason about and reject the noisy measurements it encounters due to its improved representation of the nonlinearities in the estimate uncertainty.

\subsection{Mapping with a Mobile Node}

To test the influence of mobile nodes on the network mapping problem, we introduced a mobile node into the static network. In addition to the mobile node, there were 11 other stationary nodes deployed for this experiment. Figure 5 shows the estimated node positions and path of the mobile node using our proposed method. As can be seen, the resultant laser map that is generated by overlaying the laser scans from the mobile node on top of the estimated path is very similar to the true floor plan map shown in Figure 2. Note that the map generated by overlaying the laser scans on the estimated robot path provides a good visual evaluation of the accuracy of the estimate provided by the filter. In addition to revealing any errors in estimating the mobile node's position, the map overlay also highlights errors in estimating the heading of the mobile node. It can be observed that much of the error in the resultant 


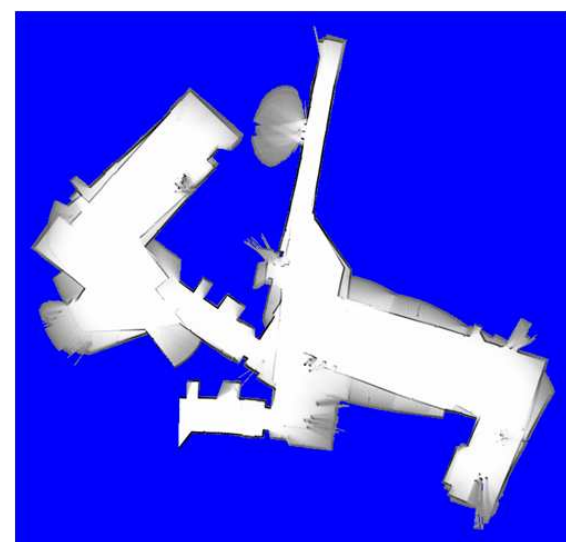

Fig. 4. The laser map generated by the scan matching algorithm within CARMEN robotics toolkit [13]. Scan matching fails due to the lack of features visible in the short-sighted laser scans ( $\max$ range $8 \mathrm{~m}$ ).

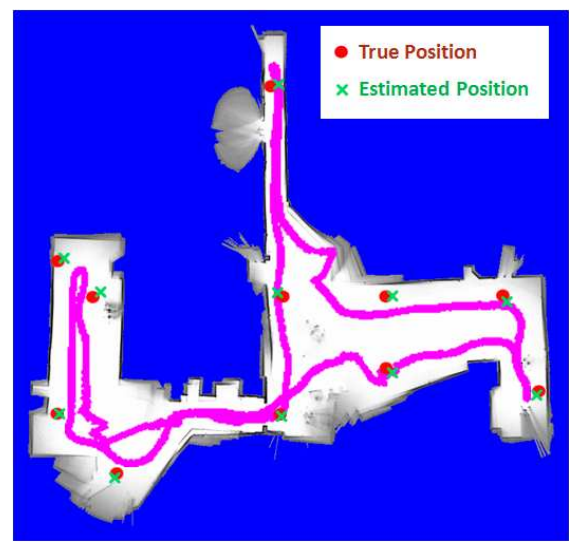

Fig. 5. The laser map generated by overlaying the laser scans from the mobile node on top of the estimated path of the mobile node using our proposed method. The proposed method assumes only two anchors, whose positions were known a priori. The resulting map looks closer to the true map and highlights the proposed method's position and heading accuracy.

\begin{tabular}{|c||c|c|}
\hline Method & Avg. Node Err. & Avg. Laser Map Err. \\
\hline \hline Dead Reckoning & - & $9.62 \mathrm{~m}$ \\
\hline Scan Matching & - & $12.27 \mathrm{~m}$ \\
\hline \hline $\begin{array}{c}\text { Static Mapping } \\
\text { (4 Anchors) }\end{array}$ & $1.89 \mathrm{~m}$ & - \\
\hline $\begin{array}{c}\text { SLAM Initialized w/ } \\
\text { Static Mapping Result }\end{array}$ & $0.92 \mathrm{~m}$ & $1.86 \mathrm{~m}$ \\
\hline $\begin{array}{c}\text { SLAM } \\
\text { (2 Anchors) }\end{array}$ & $1.19 \mathrm{~m}$ & $2.62 \mathrm{~m}$ \\
\hline $\begin{array}{c}\text { SLAM } \\
\text { (All 11 Anchors) }\end{array}$ & $0.00 \mathrm{~m}$ & $0.87 \mathrm{~m}$ \\
\hline
\end{tabular}

Table 1. Average node mapping error and average error in the corner features of the laser map generated using the mobile node's estimated position.

map (e.g. blurring of wall edges) is due to errors in estimating mobile node's heading correctly. This is because, in general, heading is difficult to estimate given range-only data. It should be noted here that the laser scans, used to generate the map in Figures 5, are only used to visualize the accuracy of the estimated position and heading of the mobile node (ie. the laser scans are not used to improved the estimate). 
Additionally, we can compare the result of our approach to the laser map generated by a simple scan matching algorithm (available in CARMEN [13]), Figure 4. The scan matching algorithm fails in this environment due to the lack of features in each scan. This is because the laser scanner had a maximum range of $8 \mathrm{~m}$ and in certain areas was only able to see one wall within the environment. Note that the use of a laser scanner with a maximum range of $8 \mathrm{~m}$ is not ideal for this environment, and comparing the result of using such a sensor (clearly unsuited for this environment) against our proposed method is most definitely unfair. However, it is also equally important to note that utilizing a laser scanner with a short range in the small environment shown here is analogous to using a laser scanner with a large range in a much larger environment. And as such, comparing the proposed ranging radio-based map against the laser scan matching based map highlights the benefits and utility of using ranging radios as a complimentary sensor to other more commonly used sensors, such as laser scanners.

Table 1 presents the numeric results comparing the proposed method against several other strategies, including an initialized version of SLAM, where the static mapping result (shown in Figure 3) is used to initialize the state of the nodes when performing SLAM with a mobile node. The node errors reported in the table above were calculated based on manually surveyed ground truth node locations. The "laser map" errors reported in the table were calculated based on extracting corner features from the estimated map and comparing it against the locations of those same features within the ground truth floor plan, supplemented with manual measurement of those corner features within the actual environment. For this environment, a total of 23 corner features and 11 node positions were examined to produce the results shown in the above table. In the cases when a corner feature was blurred within the estimated laser map, the worse case position of the corner was used. In other words, if the corner in an estimated map is blurry (i.e. the multiple laser scans of the corner are not properly aligned), the corner extracted from the laser scan that was the farthest from the true corner location was used to compute the error. As can be seen in the table, the average node position error for performing SLAM with only 2 anchors is similar to performing SLAM initialized with the static mapping solution (which used 4 anchors).

Figure 6 shows the performance of our algorithms as the number of anchors in the environment is varied. As can be expected, when the number of anchors increases, the error in the position of the nodes in the environment decreases. In particular, looking at the effect of adding a single mobile node to the network, it can be observed that the addition information provided by the odometry of the mobile node significantly helps improve the overall node mapping error. 


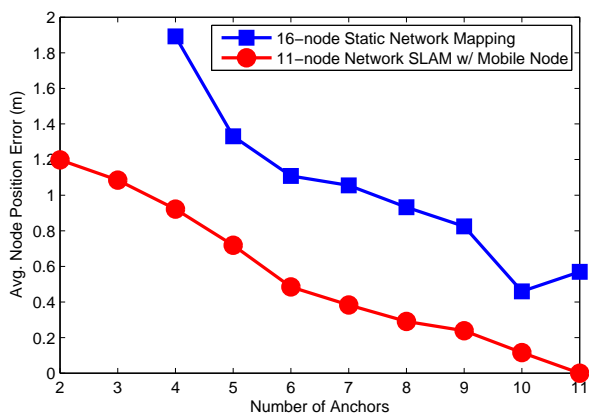

Fig. 6. Plots revealing the effects of varying the number of anchors in the environment is shown. As can be expected, in both the static network mapping case with 16-nodes (blue squares) and the 11-node network mapping case with a mobile node (red circles), as the number of anchors is increased, the over all error in mapping the node locations decreases. However, as can be seen, given the additional information from the odometry of a mobile node, the network can be better localized with much few number of anchors.

\section{Conclusions}

In this paper, we examined the problem of motion-aided network SLAM. The method we proposed offers an alternate polar parameterization that is better suited for dealing with the nonlinear measurement distributions evident in range-only data. We show that through the use of this improved parameterization, and standard measurement filtering techniques, it is possible achieve improved localization and mapping of a sparsely connected network of nodes in the presence of noisy range-only measurements. The benefits of employing range sensors and a proper representation of the state, to provide good localization and mapping results in an environment where laser scanners might fail was demonstrated. Comparing the results of our approach to laser based scan matching techniques, our experiments reveals that while the proposed range-based mapping solution has difficulty estimating heading, in some cases it offers a good complimentary solution to traditional laser based mapping techniques. Lastly, our results also revealed that by adding a single mobile

node into an otherwise static network of nodes, the overall node mapping error can be reduced considerably.

\section{Acknowledgments}

This work is supported in part by The Boeing Company. 


\section{References}

1. J. Djugash, S. Singh, G. Kantor, and W. Zhang, "Range-only slam for robots operating cooperatively with sensor networks," in IEEE Int'l Conf. on Robotics and Automation (ICRA), 2006.

2. J. Djugash, S. Singh, and B. P. Grocholsky, "Decentralized mapping of robotaided sensor networks," in IEEE International Conference on Robotics and Automation, May 2008.

3. P. Bahl and V. Padmanabhan, "Radar: An in-building RF-based user location and tracking system," in In Proc. of the IEEE Infocom 2000, Tel Aviv, Israel, March 2000.

4. N. Priyantha, A. Chakraborty, and H. Balakrishman, "The cricket location support system," in In Proc. of the 6th Annual ACM/IEEE International Conference on Mobile Computing and Networking (MOBICOM), Boston, MA, August 2000.

5. A. Smith, H. Balakrishnan, M. Goraczko, and N. B. Priyantha, "Tracking Moving Devices with the Cricket Location System," in 2nd International Conference on Mobile Systems, Applications and Services (Mobisys), Boston, MA, June 2004.

6. N. T. GmbH, "nanoloc trx transceiver (na5tr1)," Datasheet NA-06-0230-03882.00, April 2008.

7. I. Borg and P. Groenen, "Modern multidimensional scaling: theory and applications." New York: Springer, 1997.

8. D. Moore, J. Leonard, D. Rus, and S. Teller, "Robust distributed network localization with noisy range measurements," in in SenSys '04:Proc 2nd international conference on Embedded networked sensor systems. New York: ACM Press, 2004, pp. 50-61.

9. E. Olson, J. Leonard, and S. Teller, "Robust range-only beacon localization," in Proceedings of Autonomous Underwater Vehicles, 2004.

10. J. Djugash and S. Singh, "A robust method of localization and mapping using only range," in International Symposium on Experimental Robotics, July 2008.

11. C. Faloutsos and K.-I. Lin, "FastMap: A fast algorithm for indexing, data-mining and visualization of traditional and multimedia datasets," in Proceedings of the 1995 ACM SIGMOD International Conference on Management of Data, M. J. Carey and D. A. Schneider, Eds., San Jose, California, 22-25 1995, pp. 163-174.

12. J. Djugash, S. Singh, and B. Grocholsky, "Modeling mobile robot motion with polar representations," in International Conference on Intelligent Robots and Systems, October 2009.

13. M. Montemerlo, N. Roy, and S. Thrun, "Perspectives on standardization in mobile robot programming: The Carnegie Mellon navigation (CARMEN) toolkit," in Proc. IEEE/RSJ Int. Conf. Intelligent Robots and Systems, 2003, pp. 24362441. 